\title{
Rescue Balloon Pulmonary Angioplasty Under Veno-Arterial Extracorporeal Membrane Oxygenation in a Patient With Acute Exacerbation of Chronic Thromboembolic Pulmonary Hypertension
}

\author{
Makiko NaKamura, ${ }^{1}$ MD, Osahiko Sunagawa, ${ }^{1} \mathrm{MD}$, \\ Hiroyuki Tsuchiya, ${ }^{1}$ MD, Takafumi MiYara, ${ }^{1}$ MD, Yoji TABA, ${ }^{1}$ MD, Takashi Touma, ${ }^{1}$ MD, \\ Hiroshi MunaKata, ${ }^{2}$ MD, Tadao Kugai, ${ }^{2}$ MD, and Yutaka OKITA, ${ }^{3}$ MD
}

\begin{abstract}
SUMMARY
We describe a case of a 41-year-old woman with acute exacerbation of chronic thromboembolic pulmonary hypertension (CTEPH) complicated by rapidly progressive respiratory failure and right heart failure with cardiogenic shock. A computed tomography (CT) showed thrombi in the right main pulmonary artery and bilateral peripheral pulmonary arteries, and echocardiography showed right ventricular dilatation and tricuspid regurgitation, with an estimated pressure gradient of $80 \mathrm{mmHg}$. The patient was initially diagnosed with acute pulmonary thromboembolism, and thrombolytic therapy was administered. Her condition subsequently deteriorated, however, necessitating mechanical ventilation and veno-arterial extracorporeal membrane oxygenation (VA-ECMO). We performed emergency catheter-directed thrombectomy and thrombus aspiration. Pulmonary hypertension $(\mathrm{PH})$ temporarily improved, but subsequently worsened, and the patient was diagnosed with CTEPH. Pulmonary endarterectomy (PEA) was performed. After PEA, we were unable to wean the patient off VA-ECMO, and rescue balloon pulmonary angioplasty (BPA) to the middle and inferior lobe branches of the right lung was performed. Five days after BPA, the patient was removed from VA-ECMO and on the 57th day of hospitalization, she was weaned off the ventilator. The patient was discharged after 139 days of hospitalization. Rescue BPA represents a useful intervention for improving PH and weaning off VA-ECMO in a patient with acute exacerbation of CTEPH. (Int Heart J 2015; 56: 116-120)
\end{abstract}

Key words: Right heart failure, Pulmonary endarterectomy, BPA, ECMO, CTEPH

$\mathrm{C}$ hronic thromboembolic pulmonary hypertension (CTEPH) is caused by organized pulmonary thrombosis, and is considered to progress, at least in part, from acute pulmonary thromboembolism, though the exact etiology remains unknown. Five-year survival rates in patients with a mean pulmonary artery pressure (PAP) less than 30 mmHg is approximately $90 \%$; a mean PAP over $50 \mathrm{mmHg}$ confers a survival rate of approximately $10 \%$, and very poor prognosis. ${ }^{1)}$ Pulmonary endarterectomy (PEA) in patients with CTEPH can markedly decrease PAP and improve prognosis. ${ }^{2}$ In peripheral types of CTEPH, however, PEA is ineffective and associated mortality rates are high. Recently, the efficacy of balloon pulmonary angioplasty (BPA) in inoperable CTEPH patients was reported. ${ }^{3)}$ We successfully employed BPA under veno-arterial extracorporeal membrane oxygenation (VA-EC$\mathrm{MO}$ ) in a patient with acute exacerbation of CTEPH that did not improve with PEA.

\section{Case Report}

A 41-year-old woman presented at our hospital with dyspnea, edema, and weight gain. She had suffered from epilepsy since the age of 16, and at 36 had exhibited multiple lacunar infarctions on magnetic resonance imaging (MRI). She was a heavy smoker (40 cigarettes per day for 20 years), but had no family history of thromboembolic disease or blood coagulation disorders. She was taking phenytoin $100 \mathrm{mg}$ tid, valproic acid $400 \mathrm{mg}$ qid, and aspirin $100 \mathrm{mg}$ sid.

On admission, her blood pressure was 102/58 mmHg; pulse rate was 106 beats/minute; and oxygen saturation, measured by pulse oximetry, was $94 \%$ under oxygen inhalation of 3 $\mathrm{L} /$ minute. Auscultation revealed crackles in the middle and inferior right lung field, but no heart murmurs. The patient had abdominal distension, leg edema, and cold extremities. A chest radiography showed cardiomegaly, pulmonary congestion, and infiltration in the right lower lung field. An electrocardiogram showed right axis deviation and a negative $\mathrm{T}$ wave at $\mathrm{V} 1-\mathrm{V} 4$. Echocardiography demonstrated a markedly enlarged right

From the Departments of ${ }^{1}$ Cardiology, ${ }^{2}$ Cardiovascular Surgery, Okinawa Prefectural Nanbu Medical Center and Children's Medical Center, Okinawa, and ${ }^{3}$ Department of Surgery, Division of Cardiovascular Surgery, Kobe University Graduate School of Medicine, Kobe, Japan.

Address for correspondence: Makiko Nakamura, MD, Department of Cardiology, Okinawa Prefectural Nanbu Medical Center and Children's Medical Center, 118-1 Arakawa, Haebaru-cho, Shimajiri-gun, Okinawa 901-1193, Japan. E-mail: nakamuramk1979@ gmail.com

Received for publication August 3, 2014. Revised and accepted August 14, 2014.

Released in advance online on J-STAGE January 7, 2015.

All rights reserved by the International Heart Journal Association. 

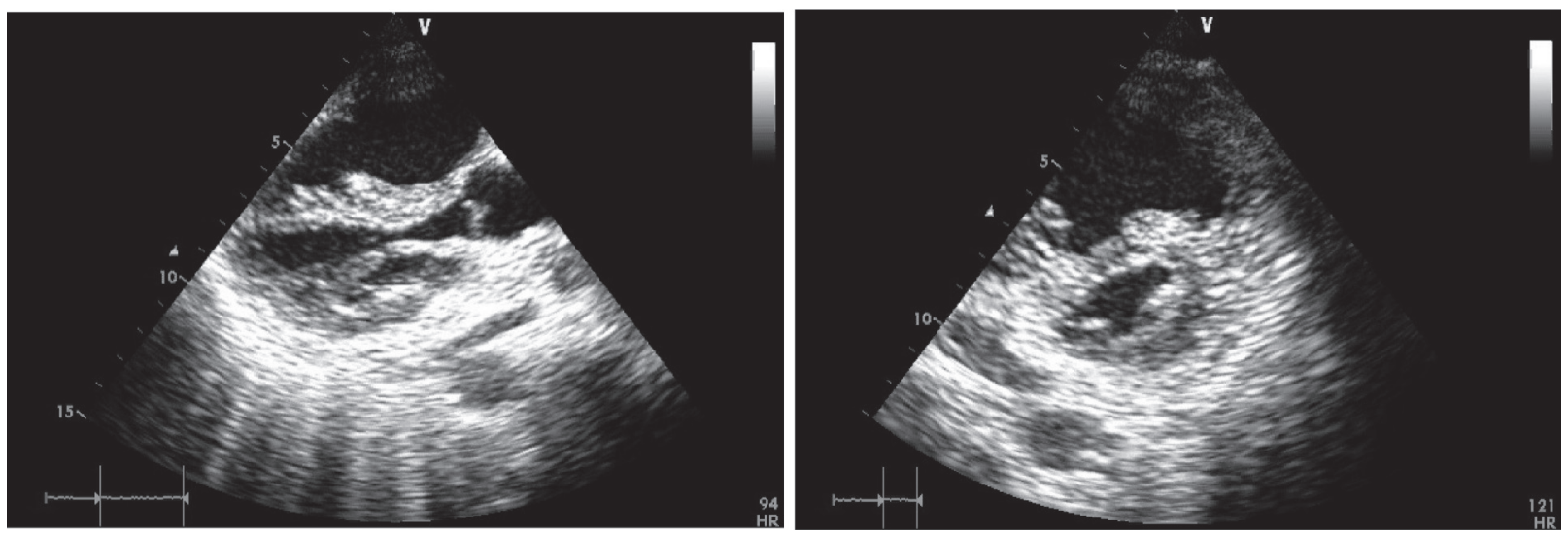

Figure 1. Echocardiography demonstrated an enlarged right ventricle and a compressed left ventricle.
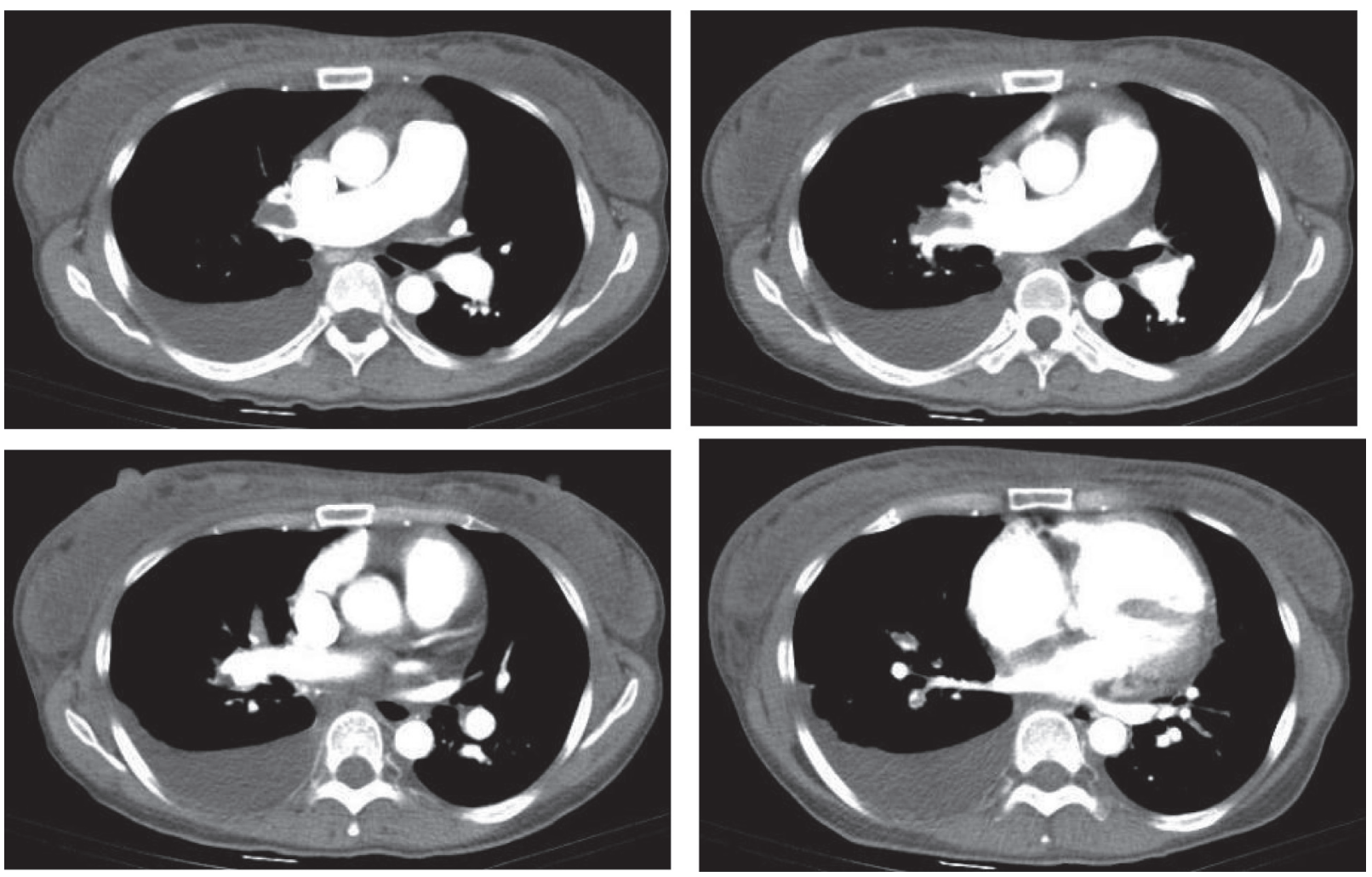

Figure 2. Contrast enhanced CT showed a right main pulmonary artery thrombus and bilateral peripheral pulmonary artery thrombi.

ventricle, tricuspid regurgitation (TR) with a peak pressure gradient of $80 \mathrm{mmHg}$, and a dilated inferior vena cava (Figure 1). Blood cell counts were roughly normal, but serum levels of aspartate transaminase, alanine transaminase, alkaline phosphatase, and lactate dehydrogenase were elevated: 73 IU/L, $133 \mathrm{IU} / \mathrm{L}, 430 \mathrm{IU} / \mathrm{L}$, and $369 \mathrm{IU} / \mathrm{L}$, respectively. Blood urea nitrogen was slightly elevated $(23 \mathrm{mg} / \mathrm{dL})$; serum creatinine and total bilirubin were normal $(0.5 \mathrm{mg} / \mathrm{dL}$ and $0.4 \mathrm{mg} / \mathrm{dL}$, respectively). Brain natriuretic peptide was elevated (1681.7 pg/ $\mathrm{mL}$ ), as were serum C-reactive protein and D-dimer values $(8.11 \mathrm{mg} / \mathrm{dL}$ and $5.3 \mu \mathrm{g} / \mathrm{mL}$, respectively). Anti-nuclear antibody was negative, and protein $\mathrm{C}$ activity was decreased by
$36 \%$, suggesting protein $\mathrm{C}$ deficiency. Blood gas analysis showed respiratory alkalosis $(\mathrm{pH} 7.503$; pCO2 $26.7 \mathrm{mmHg}$; pO2 75.2 mmHg; HCO3- $20.8 \mathrm{mmol} / \mathrm{L}$; and $\mathrm{SaO} 2$ 96.1\%, under a nasal oxygen supply of $3 \mathrm{~L} /$ minute). A contrast-enhanced computed tomography (CT) scan showed a right main pulmonary artery thrombus and bilateral peripheral pulmonary artery thrombi (Figure 2).

The patient was initially diagnosed with acute pulmonary thromboembolism, and recombinant tissue plasminogen activator (rt-PA 80,000 U; Monteplase) was administered. The intervention was ineffective, however: oxygen saturation and peak pressure gradients of TR, measured by echocardiography, 
did not improve. Central venous pressure was increased to 18 $\mathrm{mmHg}$.

On the 2nd day of hospitalization, oxygenation deteriorated gradually, and we performed tracheal intubation for ventilator support. Despite intubation and a fraction of respired oxygen $(\mathrm{FiO} 2)$ of $100 \%$, the patient's oxygenation and blood pressure continued to decline, and we decided to administer VA-ECMO. Blood pressure and oxygenation subsequently stabilized, and we performed emergency pulmonary artery angiography. A pulmonary angiogram revealed clots in the center of the right middle and inferior lobe branches. We performed catheter-directed thrombectomy to crush the clots with a pigtail catheter. After crushing of the thrombi, blood flow to the right upper lobe branch was slightly improved, and pulse infusion thrombolysis (PIT) was performed with 240,000 U urokinase, and thrombus aspiration with a Judkins right (JR) 4.0 catheter. Finally, we measured PAP with an indwelling SwanGanz catheter. On VA-ECMO with a support flow of $2.2 \mathrm{~L} /$ minute, PAP was 45/29 $\mathrm{mmHg}$ with $34 \mathrm{mmHg}$ of a mean pressure, pulmonary capillary wedge pressure (PCWP) was 10 $\mathrm{mmHg}$, and right atrium pressure (RAP) was $8 \mathrm{mmHg}$.

On the third day after admission, PAP was 41/32 (38) mmHg under VA-ECMO of $2.2 \mathrm{~L} /$ minute, and administration of inhaled nitric oxide (NO), and intravenous epoprostenol and milrinone was initiated. On the following day, PAP was raised to $90 / 40$ (60) $\mathrm{mmHg}$ under VA-ECMO of $3.0 \mathrm{~L} /$ minute, and we were unable to withdraw VA-ECMO.

The patient was subsequently diagnosed with CTEPH, and we consulted cardiac surgeons on the surgical indications for PEA. Because of the large thrombi in the right main pulmonary artery, PEA was performed on the 7th day of hospitalization, but as there were few organized thrombi in the central pulmonary artery, her condition was classified as peripheral CTEPH.

On the day after PEA, the RAP was $18 \mathrm{mmHg}$, and PAP was $41 / 28$ (33) $\mathrm{mmHg}$ on VA-ECMO of $3.0 \mathrm{~L} /$ minute. However, on the 9th day of hospitalization, the urine volume decreased, and continuous hemodiafiltration (CHDF) was initiated.

On the 10th day, a mediastinal hematoma was discovered on a contrast-enhanced CT scan; we reoperated on the following day and the hematoma was drained.

Following the second operation, mean PAP was raised to $50 \mathrm{mmHg}$ on VA-ECMO of $1.0 \mathrm{~L} /$ minute, and we consequently administered sildenafil (60 mg/day), ambrisentan $(2.5 \mathrm{mg} /$ day), and epoprostenol (10 ng/kg/minute). Although we attempted to wean the patient off VA-ECMO, we were unsuccessful.

Since medical and surgical interventions had been ineffective, we performed emergent BPA through the left femoral vein on the 13th day of hospitalization. A guiding catheter (6Fr, 100-cm JR 4.0; AXESS, ASAHI INEC CO LTD, Nagoya, Japan) was inserted into the main pulmonary artery using a 0.035-inch swan-neck hydrocoated guidewire (Swan-Excel, Fellow Co. Ltd, Toyohashi, Japan); right and left branches of the pulmonary artery were manipulated using the guidewire. A pulmonary angiogram showed an improvement in pulmonary blood flow in the apical segmental artery (A1), posterior segmental artery (A2), anterior segmental artery (A3), and lateral segmental artery (A4), but stenosis persisted in the peripheral pulmonary artery: stenosis was evident in the anterior basal segmental artery (A8), and total occlusion of the posterior basal segmental artery (A10) was observed. We performed BPA using a 0.014-inch floppy guidewire (Runthrough Peripheral, TERUMO, Japan) with careful and gentle manipulation of the occlusion in A10; vessel diameter and characteristics of stenosis were estimated by intravascular ultrasound (IVUS; EagleEye, Volcano Japan, Tokyo), and pulmonary stenosis was dilated using a 2.0-mm-diameter balloon (Jackal RX, Kaneka Medical Products, Osaka, Japan). Blood flow was subsequently restored. This procedure was repeated in the middle segmental artery (A5), and blood flow in A4 and A5 was successfully restored (Figure 3). After BPA, a chest radiograph showed a lung infiltration shadow in the right lower lung, but arterial oxygenation was maintained under extracorporeal lung support.

On the 17th day of hospitalization, the patient was weaned off VA-ECMO.

She was removed from CHDF on day 41 , and the mechanical ventilator on day 57 .

Right heart catheterization was performed on day 124 . RAP was $4 \mathrm{mmHg}$; right ventricular end diastolic pressure was $5 \mathrm{mmHg}$; PAP was 38/12 (23) $\mathrm{mmHg}$; and PCWP was 7 $\mathrm{mmHg}$. Cardiac output and cardiac index were $5.48 \mathrm{~L} /$ minute and $4.03 \mathrm{~L} / \mathrm{minute} / \mathrm{m}^{2}$, respectively. Pulmonary vascular resistance was 340 dynes $/ \mathrm{sec} / \mathrm{cm}^{5}$.

Lung perfusion scintigraphy showed multiple defects in the middle field of the right lung and inferior field of the left lung on day 126 (Figure 4).

An electrocardiogram showed no right axis deviation, and the $\mathrm{T}$ wave at V3-4, which had previously been negative, was positive, indicating attenuation of right heart overload. There were no signs of right heart overload on physical examination. The patient was discharged 139 days after admission.

Follow-up echocardiography at 1 month after discharge showed no right ventricular dilatation, and the peak pressure gradient of TR was $28 \mathrm{mmHg}$, indicative of a systolic PAP of $33-38 \mathrm{mmHg}$.

\section{DisCUSSION}

Chronic thromboembolic pulmonary hypertension (CTEPH) is one of the most prevalent forms of pulmonary hypertension $(\mathrm{PH})$, arising in approximately $4 \%$ of patients after acute pulmonary embolism. ${ }^{4)}$ There is now accumulating evidence, however, that CTEPH may also develop in the absence of previous pulmonary embolism. ${ }^{4)}$ PEA is thought to be the most effective treatment for CTEPH, especially in cases of proximal organized thrombi, where it may be curative. ${ }^{5)}$ The indication for PEA depends on the location of organized thrombi, the severity of $\mathrm{PH}$, patient age, and co-morbidities; distal or peripheral obstructions may not be suitable for surgical intervention, and an effective treatment may not be identified for non-responsive patients with distal-type CTEPH that persists after PEA.

For CTEPH patients in whom PEA is not indicated, medial therapy, such as endothelin receptor antagonists or phosphodiesterase (PDE)-5 inhibitors, can be administered. Condliffe, et al reported improved survival in patients treated with endothelin receptor antagonists and PDE-5 inhibitors. However, the effectiveness of medical intervention was limited, and 

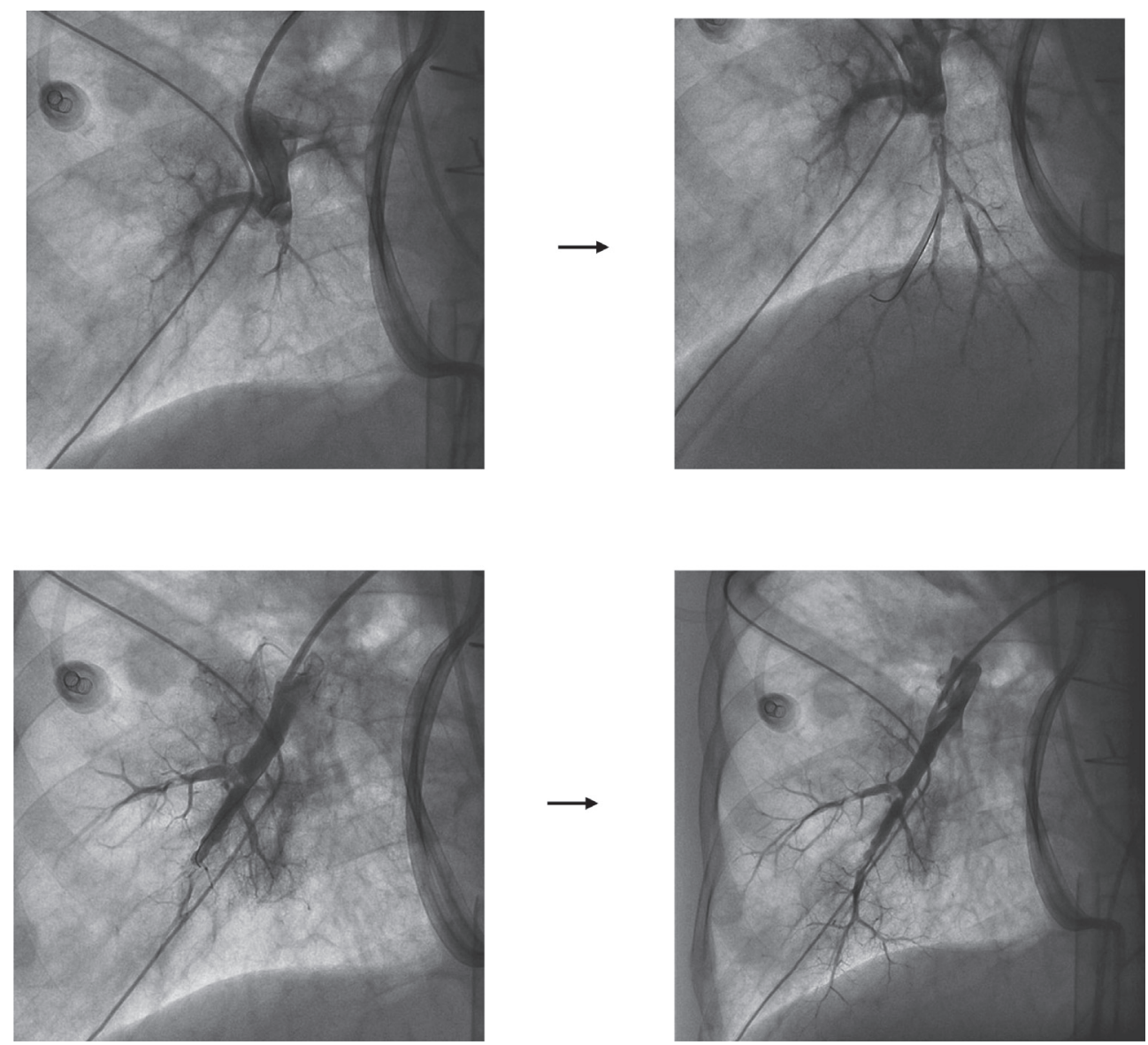

Figure 3. We performed BPA.

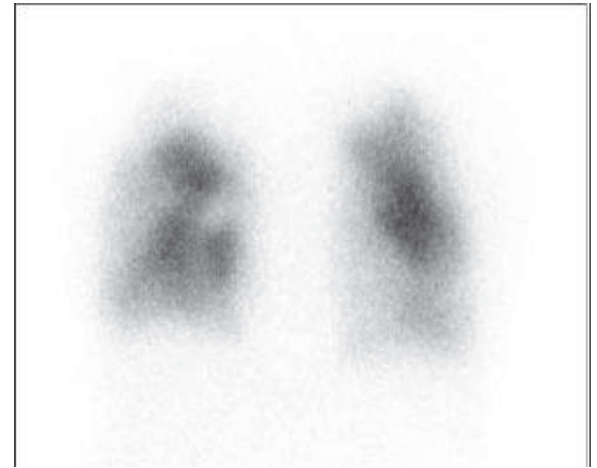

Figure 4. Lung perfusion scintigraphy showed multiple defects in the middle field of the right lung and inferior field of the left lung.

the hemodynamic improvement was considered to be inferior to that achieved with PEA. $\left.{ }^{6}\right)$ Recently, riociguat-a soluble guanylyl cyclase stimulator-was developed as a promising new drug for pulmonary artery hypertension (PAH). In a randomized trial, riociguat significantly improved 6-minute walking distance, pulmonary vascular resistance, and other clinical outcomes in patients with CTEPH. ${ }^{7}$ Its impact on acute exacerbation and survival in patients with CTEPH, however, remains unknown, and its clinical efficacy and survival benefit remain to be tested in a randomized controlled trial.

In a recent meta-analysis of 11 studies (with a total of 269 CTEPH patients), administration of bosentan, an endothelin receptor antagonist, was associated with improved pulmonary hemodynamics and exercise capacity. ${ }^{8}$ Nishimura, et al reported improved survival in patients with CTEPH treated with bosentan and sildenafil. ${ }^{9)}$ However, with the exception of riociguat, medications for PAH treatment have been used off-label in patients with CTEPH who are inoperable or who exhibit persistent PH after PEA; such drugs have also been used as therapeutic bridges before PEA. ${ }^{10)}$

The patient described in this case had central and distal thrombi in the pulmonary arteries, and cardiac surgeons initially performed emergency PEA under VA-ECMO. However, hemodynamic improvement after PEA was limited; residual peripheral thrombi remained, and we were unable to wean the patient off VA-ECMO, despite treatment with modern PAH therapy (ie, a continuous infusion of epoprostenol-a PDE-5 inhibitor-and an endothelin receptor antagonist). Although riociguat may be effective in patients with CTEPH, it was unfortunately not licensed for clinical use in Japan at the time.

The BPA was first reported in 1988 by Voorburg, et al, who described its use in a 30-year-old man with PH after pulmonary thromboembolism. ${ }^{11)}$ In 2001, the efficacy of BPA was tested in 18 patients with CTEPH by Feinstein, et al. ${ }^{12)} \mathrm{Al}-$ though a significant improvement in hemodynamics and exer- 
cise tolerance was achieved, the magnitude of change was inferior to that achieved with PEA. Moreover, 11 of the 18 patients developed reperfusion pulmonary edema (RPE); 3 required mechanical ventilation, and 2 patients $(11.1 \%)$ died. The mortality rate of BPA was not superior to that of PEA. The researchers concluded that BPA is a labor-intensive approach to management that confers potentially lethal complications, though their results were considered sufficiently promising to justify the indication for BPA in inoperable CTEPH patients.

Mizoguchi, et al subsequently refined the BPA procedure with appropriate balloon sizing and staged procedures to reduce the risk of pulmonary reperfusion injury. ${ }^{3)}$ This refined procedure was found to improve the clinical status and hemodynamics of inoperable CTEPH patients, with a low mortality rate. The refined BPA procedure may be considered as a viable therapeutic approach for patients with inoperable CTEPH.

In line with the procedure developed by Mizoguchi, et al, we performed BPA using a 0.014-inch soft-tip guidewire, with IVUS and an undersized balloon to the reference diameter of the pulmonary arteries for BPA, in order to prevent pulmonary artery injury and pulmonary edema. Operators must be aware of bleeding tendency in patients under VA-ECMO with anticoagulant therapy, which can facilitate pulmonary hemorrhage or extravasation at the balloon site, precipitating pulmonary ede$\mathrm{ma}$; in this case, hemodynamic stability was successfully achieved with VA-ECMO.

BPA has also been used to stabilize the hemodynamic status of CTEPH patients who are non-responsive to PEA. ${ }^{13)}$ BPA may be performed multiple times in patients who have unresolved PH after PEA. In this case, the patient's clinical status was improved following additional BPA after PEA, and in cases of CTEPH with both central and peripheral thrombi, additional BPA may be effective.

Inami, et al proposed an index to reflect both the change in angiographic flow and the baseline severity of $\mathrm{PH}$ due to CTEPH. ${ }^{14)}$ According to this report, the index is related to the development of RPE and both the change in angiographic flow and the baseline severity of PH. To avoid vessel injury and widespread pulmonary edema, we performed BPA using a small balloon ( $2.0 \mathrm{~mm}$ in diameter), and limited the area within the right middle or lower lobes.

Tsuji, et al reported rescue BPA in a rapidly deteriorating CTEPH patient with liver failure and refractory infection. ${ }^{15}$ ) They suggested that BPA may be the only effective therapeutic option for deteriorating, inoperable patients. Our patient also improved after rescue BPA under mechanical cardiopulmonary support, following initial PEA.

Our case also suggests that emergency BPA may be a rescue option for CTEPH patients with peripheral thrombi, who do not respond to PEA, or for whom PEA is not indicated due to comorbid conditions, such as accelerating $\mathrm{PH}$ and rapidly progressive heart failure, especially when mechanical cardiopulmonary support is required.

\section{REFERENCES}

1. Riedel M, Stanek V, Widimsky J, Prerovsky I. Longterm followup of patients with pulmonary thromboembolism. Late prognosis and evolution of hemodynamic and respiratory deta. Chest 1982; 81: 151-8.

2. Thistlethwaite PA, Mandani M, Jamieson SW. Outcomes of pulmonary endarterectomy surgery. Semin Thorac Cardiovasc Surg 2006; 18: 257-64.

3. Mizoguchi H, Ogawa A, Munemasa M, Mikouch H, Ito H, Matsubara H. Refined balloon pulmonary angioplasty for inoperable patients with chronic thromboembolic pulmonary hypertension. Circ Cardiovasc Interv 2012; 5: 748-55.

4. Pengo V, Lensing AW, Prins MH, et al. Incidence of chronic thromboembolic pulmonary hypertension after pulmonary embolism. N Engl J Med 2004; 350: 2257-64.

5. Galiè N, Hoeper MM, Humbert M, et al. Guidelines for the diagnosis and treatment of pulmonary hypertension: the Task Force for the Diagnosis and Treatment of Pulmonary Hypertension of the European Society of Cardiology (ESC) and the European Respiratory Society (ERS), endorsed by the International Society of Heart and Lung Transplantation (ISHLT). Eur Heart J 2009; 30: 2493537.

6. Condliffe R, Kiely DG, Gibbs JS, et al. Improved outcomes in medically and surgically treated chronic thromboembolic pulmonary hypertension. Am J Respir Crit Care Med 2008; 177: 1122-7.

7. Ghofrani HA, D'Armini AM, Grimminger F, et al. Riociguat for the treatment of chronic thromboembolic pulmonary hypertension. N Engl J Med 2013; 369: 319-29.

8. Becattini C, Manina G, Busti C, Gennarini S, Agnelli G. Bosentan for chronic thromboembolic pulmonary hypertension: findings from a systematic review and meta-analysis. Thromb Res 2010; 126: e51-6. (Review)

9. Nishimura R, Tanabe N, Sekine A, et al. Survey for the prognosis of medically-treated chronic thromboembolic pulmonary hypertension. MHLW Respiratory Failure Research Group Report in 2011 2012: 241-3. (Japanese)

10. Fedullo P, Kerr KM, Kim NH, Auger WR. Chronic thromboembolic pulmonary hypertension. Am J Respir Crit Care Med 2011; 183: 1605-13. (Review)

11. Voorburg JAI, Cats VM, Buis B, Bruschke AV. Balloon angioplasty in the treatment of pulmonary hypertension caused by pulmonary embolism. Chest 1988; 94: 1249-53.

12. Feinstein JA, Goldhaber SZ, Lock JE, Ferndandes SM, Landzberg MJ. Balloon pulmonary angioplasty for treatment of chronic thromboembolic pulmonary hypertension. Circulation 2001; 103: $10-3$.

13. Satoh K, Fukumoto Y, Sugimura K, et al. A case of chronic thromboembolic pulmonary hypertension that balloon pulmonary angioplasty was effective for residual pulmonary hypertension after pulmonary endarterectomy. J Cardiol Suppl 2010; 5: 420. (Japanese)

14. Inami $\mathrm{T}$, Kataoka M, Shimura $\mathrm{N}$, et al. Pulmonary edema predictive scoring index (PEPSI), a new index to predict risk of reperfusion pulmonary edema and improvement of hemodynamics in pertaneous transluminal pulmonary angioplasty. JACC Cardiovasc Interv 2013; 6: 725-36

15. Tsuji A, Ogo T, Demachi J, et al. Rescue balloon pulmonary angioplasty in a rapidly deteriorating chronic thromboembolic pulmonary hypertension patient with liver failure and refractory infection. Pulm Circ 2014; 4: 142-7. 